\title{
Surprise et magie de la surprise
}

Essai d'interprétation psycho-phénoménologique

\section{Philippe Cabestan}

\section{(2) OpenEdition}

\section{Journals}

Édition électronique

URL : http://journals.openedition.org/alter/427

DOI : $10.4000 /$ alter.427

ISSN : 2558-7927

Éditeur :

Association ALTER, Archives Husserl (CNRS-UMR 8547)

\section{Édition imprimée}

Date de publication : 1 décembre 2016

Pagination : 107-122

ISBN : 978-2-9550449-2-6

ISSN : 1249-8947

Référence électronique

Philippe Cabestan, «Surprise et magie de la surprise », Alter [En ligne], 24 | 2016, mis en ligne le 01 décembre 2017, consulté le 02 mai 2019. URL : http://journals.openedition.org/alter/427 ; DOI : 10.4000/alter.427 


\title{
SURPRISE ET MAGIE DE LA SURPRISE ESSAI D'INTERPRÉTATION PSYCHO-PHÉNOMÉNOLOGIQUE
}

\author{
Philippe Cabestan \\ Pour Guy Risbec
}

Notre propos sera ici des plus modestes dans la mesure où, adoptant un point de vue psychologique, nous ne chercherons pas à conférer à la surprise une signification "profonde»- à la manière dont Heidegger reconnaît à l'angoisse ou à l'ennui une dimension proprement ontologique ${ }^{1}$. Aussi, et avant même d'en élaborer rigoureusement le concept, convient-il de distinguer la surprise et l'étonnement. On sait la place éminente que lui réserve la philosophie antique $^{2}$. Comme le rappelle Aristote au début de La Métaphysique, l'étonnement est étroitement lié au désir de connaître et c'est à cette disposition humaine - voire plus qu'humaine - que l'on doit la philosophie ${ }^{3}$. De son côté, la surprise frappe les hommes à l'improviste,

\footnotetext{
${ }^{1}$ On pourrait, par exemple, interroger la dimension de «sur-prise » de l'événement au sens où l'événement suspend toute prise. Ainsi Claude Romano propose-t-il de distinguer entre une surprise qui est «le simple choc en retour d'une prévision contredite par l'arrivée d'un fait inattendu » et la sur-prise en tant que « saisissement devant et par ce qui passe nécessairement toute attente", alias l'événement en son sens événemential. Cf. C. Romano, L'événement et le monde, Paris, PUF, 1998, p. 65 ; C. Romano, L'événement et le temps, Paris, PUF, 1999, p. 221 et sq. 2 Platon, Théétète, 155 d, trad. Léon Robin, Paris, Gallimard, « La Pléiade », 1950, t. II, p. 16.

3 «C'est, en effet, l'étonnement qui poussa, comme aujourd'hui, les premiers penseurs aux spéculations philosophiques ", Aristote, Métaphysique, A, 2, 982 b, 10-15, trad. J. Tricot, Paris, Vrin, 1970, p. 16-17. Cependant, distinguer entre l'étonnement (Verwunderung) et la surprise (Überraschung) ne va pas de soi. Aristote évoque également l'étonnement que suscite un simple spectacle dont les marionnettes semblent se mouvoir d'elles-mêmes (Métaphysique, A, 2, 983a, 14), et Annie Hourcade Sciou note que dans la Poétique d'Aristote thaumaston et ekplêxis sont également susceptibles d'être traduits par le français "surprise». Cf. A. Hourcade Sciou, «La surprise dans la poétique d'Aristote", N. Depraz et C. Serban (éd.), La Surprise à l'épreuve des langues, Paris, Hermann, 2015, p. 141. En revanche, à la fin de sa conférence de 1929, Heidegger rapproche l'angoisse de l'étonnement et, si l'on peut dire, lui redonne ses lettres de noblesse métaphysique: "C'est uniquement parce que le rien est manifeste au fond de l'être-là que peut
} 
les désarçonne, les surprend de manières et dans des situations les plus diverses. Ainsi nos vaillants soldats sont-ils attaqués par surprise alors qu'Alice rencontre un surprenant lapin blanc qui court après le temps ${ }^{4}$ et que les Juifs pendant la fête des Tentes sont «surpris » par l'enseignement du Christ, « lui qui n'a pas étudié » ${ }^{5}$. Mais cette déroute de l'anticipation mise à part, que peut bien nous apporter une étude de la surprise? Dans l'Esquisse d'une théorie des émotions, Sartre, de fait, n'en dit mot. Contrairement à Paul Ricœur qui lui accorde, en raison de sa simplicité et à la suite de Descartes, une place privilégiée ${ }^{6}$, Sartre lui préfère des émotions telles que la joie, la tristesse, la peur ou encore la colère.

Cependant, ne pourrait-on pas étendre à la surprise ce que l'Esquisse nous dit d'une manière générale de l'émotion ? Ne serait-ce pas une bonne manière de mettre à l'épreuve une conception de l'émotion séduisante à bien des égards ne serait-ce qu'en raison de son originalité ? Deux éléments nous poussent en ce sens : d'une part, dans une note de la conclusion de l'Esquisse, Sartre exprime le souhait que son travail permette "d'amorcer des études monographiques complètes de la joie, de la tristesse, etc. ${ }^{7}$. D'autre part et surtout, il nous semble que la surprise, quand bien même elle ne serait pas une émotion stricto sensu, partage avec l'émotion telle que Sartre l'envisage une détermination fondamentale, que l'on retrouve dans l'horreur et l'admiration, et qui n'est autre que son caractère magique. De ce point de vue, comme nous voudrions le montrer, la surprise et, plus exactement, le moment de la surprise est ce qu'on serait tenté $\mathrm{d}^{\prime}$ appeler un instant magique. Afin de justifier et d'approfondir cette affirmation, nous voudrions tout d'abord préciser la perspective qui sera ici la nôtre; rappeler brièvement la conception sartrienne de l'émotion; nous demander si la surprise est bien en ce sens une

venir sur nous la pleine étrangeté (Befremdlichkeit) de l'étant. Ce n'est que si l'étrangeté de l'étant nous presse que celui-ci éveille et appelle à soi l'étonnement (Verwunderung). Ce n'est que sur le fond de l'étonnement - c'est-à-dire de la manifestation du rien - que surgit le "pourquoi ?" ", M. Heidegger, Qu'est-ce que la métaphysique?, trad. Roger Munier, Paris, L'Herne, 1983, p. 56.

${ }^{4} \mathrm{C}$. Serban, «La surprise comme mise en question de l'espace logique. Remarque sur la construction narrative d'Alice's Adventures in Wonderland ", La Surprise à l'épreuve des langues, op. cit., p. 241.

${ }_{5}^{5}$ Évangile selon saint Jean, 7, 15, Nouveau Testament, Paris, Les Éditions du Cerf, 1972, p. 309 ; Y. Millou, "Surprise et Parole : les verbes de la surprise dans le Nouveau Testament ", La Surprise à l'épreuve des langues, op. cit., p. 177 et $s q$.

${ }^{6}$ J.-P. Sartre, Esquisse d'une théorie des émotions, Paris, Hermann, 2010 (en abrégé : Esquisse), nous recommandons cette dernière édition du texte de Sartre, qui comprend une substantielle préface d'Arnaud Tomès. P. Ricœur, Philosophie de la volonté, t. 1, Le volontaire et l'involontaire, Paris, Aubier, 1950, p. 238.

${ }^{7}$ Esquisse, p. 65, note 1. 
émotion ; et, enfin, mettre à l'épreuve cette conception de la surprise à partir d'une célèbre coupe de cheveux que nous emprunterons à l'autobiographie de Sartre, Les Mots. Ce faisant, nous tenterons de préciser le statut d'une psychologie phénoménologique.

\section{Phénoménologie, psychologie et naturalisme}

Notre propos entend donc se tenir dans les limites d'une psychologie phénoménologique. Qu'est-ce à dire ? La réponse, à vrai dire, ne va nullement de soi. On sait que Husserl, tout au long de son œuvre et dès ses premiers travaux sur le psychologisme, ne cesse de s'interroger sur le statut de la psychologie, mettant par la suite « en parallèle » la phénoménologie transcendantale et la psychologie.

En rupture avec la psychologie expérimentale de son temps, que représente un Wilhelm Wundt ${ }^{8}$, Husserl a en effet le projet - notamment dans ses cours de $1925^{\circ}$ - d'établir les bases, grâce à la phénoménologie, d'une nouvelle psychologie. Cette psychologie dite alors phénoménologique serait dégagée du modèle expérimental de la science physique, dégagée de ce naturalisme que Husserl définit comme une conception qui ne "voit rien qui ne soit à ses yeux nature et avant tout nature physique ${ }^{10}$. Corrélat de l'attitude naturelle, l'objet de la psychologie phénoménologique n'est autre que la subjectivité intentionnelle, mieux: la subjectivité intentionnelle en tant que mondanéisation de la subjectivité transcendantale. Cette dernière opération, comme le montrent de manière particulièrement détaillée les Recherches pour la constitution ${ }^{11}$, peut à vrai dire s'accomplir sous une double modalité. Elle peut prendre, en effet, la forme d'une spiritualisation ou d'une naturalisation : dans le premier cas la subjectivité intentionnelle est appréhendée en tant qu'esprit, dans le second en tant qu'âme. Pourtant, comme l'envisage Husserl luimême à plusieurs reprises, une psychologie véritablement phénoménologique ne devrait-elle pas au fond, sous la dénomination de "psychologie transcendantale » ${ }^{12}$, se confondre avec la phénoménologie transcendantale? L'une et l'autre ne partagent-elles pas sinon

\footnotetext{
${ }^{8}$ W. Wundt, Eléments de psychologie physiologique, trad. É. Rouvier, Paris, Alcan, 1886.

${ }_{9}^{9}$ E. Husserl, Psychologie phénoménologique, trad. Ph. Cabestan, F. Dastur, N. Depraz, A. Mazzu, Paris, Vrin, 2001.

${ }^{10}$ E. Husserl, La Philosophie comme science rigoureuse, trad. M. de Launay, Paris, PUF, 1989, p. 18.

${ }^{11}$ E. Husserl, Recherches phénoménologiques pour la constitution, trad. É. Escoubas, Paris, PUF, 1982.

${ }^{12}$ L'expression revient de loin en loin dans l'œuvre de Husserl et apparaît déjà dans les Problèmes fondamentaux de la phénoménologie, trad. J. English, Paris, PUF, 1991, p. 169.
} 
les mêmes préoccupations du moins le même « objet »: la subjectivité intentionnelle dont le véritable dévoilement est subordonné à la réduction transcendantale?

S'interrogeant à son tour sur le statut d'une psychologie phénoménologique, Sartre propose de distinguer nettement entre la phénoménologie et la psychologie tout en appuyant celle-ci sur celle-là. Dans une perspective relativement fidèle à Husserl, l'Esquisse rappelle tout d'abord que les faits psychiques ne sont jamais premiers et que la psychologie ne saurait être au commencement car elle suppose la mise au jour par la réflexion des structures de la conscience transcendantale - que nous livre, en 1943, L'Être et le néant. De ce point de vue, la conscience transcendantale est une conscience préréflexive ou pour-soi dont l'existence de fait ou facticité est le résultat d'une néantisation première de l'en-soi. Tel est le fondement ontophénoménologique d'une liberté en situation dont les vécus ou faits psychiques sont «dans leur structure essentielle les réactions de l'homme contre le monde ». Dans le prolongement de ces considérations, il est alors possible d'étudier l'émotion en psychologue, c'està-dire l'émotion comme un «type organisé de conscience »13, distinct aussi bien de la conscience perceptive que de la conscience imageante ou de la conscience volitive. Comme nous allons le voir, cette conception de la psychologie offre à vrai dire un avantage sensible quoique discutable.

En effet, contrairement à Husserl, l'Esquisse interdit absolument toute forme de naturalisme psychologique, c'est-à-dire de réification des vécus psychiques sous la forme d'états psychiques dont la succession, sur le modèle des phénomènes physiques, relèverait d'un enchaînement de causes et d'effets. Alors que la conception husserlienne de l'âme, dont la constitution résulte de la naturalisation de la subjectivité transcendantale, admet la légitimité d'une approche de la vie psychique en termes de causalité «psycho-physique ou physiopsychique ${ }^{14}$, la psycho-phénoménologie sartrienne, parce qu'elle

\footnotetext{
${ }^{13}$ Esquisse, p. 13-14.

${ }^{14} \mathrm{E}$. Husserl, Recherches phénoménologiques pour la constitution, op. cit., p. 194. Il faut préciser que Husserl tient à maintenir une distance ontologique entre la réalité matérielle et la réalité psychique. C'est pourquoi il refuse en toute rigueur d'appliquer à l'âme le concept de causalité. Il écrit à ce propos: "Si nous nommons causalité ce rapport de dépendance, fonctionnel ou légal, qui est le corrélat de la constitution des propriétés persistantes d'un réal persistant du type nature, alors il ne peut absolument pas être question de causalité en ce qui concerne l'âme ". En effet, les choses matérielles "sont des réalités sans histoire " qui ignorent cette dépendance caractéristique de l'âme par rapport à son propre passé et par rapport aux autres réalités psychiques. Husserl en conclut que la réalité psychique a « une quasi-nature et une quasi-causalité », ibid., p. 196-197.
} 
reconnaît d'emblée la spécificité du mode d'être de la conscience, tourne résolument le dos à la tentation de chosifier le psychisme. De ce point de vue, la théorie périphérique de l'émotion perd immédiatement sa légitimité tout en offrant un bon exemple, parmi tant $\mathrm{d}^{\prime}$ autres, de la manière dont une psychologie naturaliste peut se fourvoyer. Soutenir, en effet, qu'une mère est triste parce qu'elle pleure ne fait que renverser l'ordre causal de la théorie intellectualiste : une mère pleure parce qu'elle est triste ${ }^{15}$. Dans le premier cas, l'état intime est l'effet dont les troubles physiologiques sont la cause; dans le second, l'état intime est la cause dont les troubles physiologiques sont les effets. Mais dans un cas comme dans l'autre, au lieu d'être ressaisie à partir de l'être de la réalité humaine, l'émotion est assimilée à un phénomène naturel au sens naturaliste du terme. La description de l'émotion est manquée. Il convient donc de reprendre la description de l'émotion en tant qu'elle est un type de conscience présentant une structure intentionnelle spécifique. Non pas, naturellement, pour en éliminer abstraitement la dimension corporelle mais, bien au contraire, pour en ressaisir adéquatement la signification au sein même de la conduite intentionnelle qui la lui donne.

On pourrait objecter que, en rejetant toute forme de causalité, une telle psychologie se place délibérément en marge de la recherche scientifique et, en particulier, des travaux relatifs au fondement neuronal de la vie affective. Or, à l'instar de Husserl, la psychologie phénoménologique ne doit-elle pas reconnaître la légitimité d'une certaine forme de causalité physio-psychique et, partant, d'un certain naturalisme ? Certes, dans son dialogue avec le neurobiologiste JeanPierre Changeux, l'auteur de L'Homme neuronal ${ }^{16}$, Paul Ricœur regrette l'usage "non critique » par les neurosciences de «la catégorie de causalité dans le passage du neuronal au psychique ${ }^{17}$. Mais il n'en admet pas moins une corrélation entre le cerveau et le psychisme qu'il tente d'appréhender à partir de la catégorie aristotélicienne de cause matérielle ou cause substrat: le cerveau serait alors le substrat ou cause substrat du vécu, au sens limitatif, écrit-il, de la cause sine qua non de ce qui est vécu. Cependant, précise Ricœur, soutenir que le cerveau est la cause substrat de la pensée $\mathrm{n}^{\prime}$ autorise pas à affirmer que tel complexe neuronal produit tels effets

\footnotetext{
${ }^{15}$ Esquisse, p. 11

${ }^{16}$ J.-P. Changeux, L'Homme neuronal, Paris, Fayard, 1983.

17 J.-P. Changeux, P. Ricœur, La Nature et la règle. Ce qui nous fait penser, Paris, Odile Jacob, 1998, p. 54-55.
} 
mentaux. Ce serait substituer, en termes aristotéliciens, la cause efficiente à la cause matérielle. En outre, Ricœur accorde volontiers qu'il reste insatisfait par une telle approche du problème et «que ce concept de substrat ne constitue qu'un cache-misère ${ }^{18}$. Mais on peut penser avec Sartre que le problème que se pose Ricœur est tout simplement " un faux problème ${ }^{19}$. Pour la simple raison que le corps objet ou corps pour autrui, qui est exploré par la physiologie comme par la génétique ou les neurosciences, et le corps vécu ou corps pour soi appartiennent à deux plans ontologiques rigoureusement incommunicables, ce qui exclut entre eux toute forme de causalité20. Cela ne signifie naturellement pas que l'exploration du corps pour autrui est sans valeur scientifique mais simplement qu'elle demeure et demeurera toujours extérieure à la psychologie phénoménologique en tant que telle. Par la suite, une psychologie phénoménologique de la surprise ne saurait faire abstraction de la dimension corporelle de la surprise mais doit délibérément laisser de côté ce que prétendrait lui enseigner à ce propos la neurophysiologie pour lui préférer ce que lui dévoile la réflexion.

\section{L'émotion en tant que conduite magique}

Dans ce qu'on appelle vaguement et de manière particulièrement confuse l'affectivité, Sartre opère trois distinctions décisives qui ouvrent, semble-t-il, la possibilité d'un nouveau traité des «passions ». La première, exposée dès La Transcendance de l'ego, concerne l'affectivité en tant que vécu et l'affectivité en tant qu'état: la compréhension et la description de celle-là relèvent de la psychologie phénoménologique, l'explication de celle-ci de la psychologie tout court ou psychologie non phénoménologique qui a pour objet la psyché. Il convient en effet de ne pas confondre le vécu de conscience en tant que donnée immanente et absolument indubitable de la réflexion pure, et le sentiment en tant qu'objet transcendant qui relève d'une sorte de réification du vécu par la réflexion impure. Une deuxième distinction se rapporte à la structure intentionnelle de

\footnotetext{
18 Idem.

${ }^{19}$ Nous reprenons l'expression que Sartre utilise à propos du problème de la vision renversée : comment pouvons-nous redresser les objets qui se peignent renversés sur notre rétine? J.-P. Sartre, L'Être et le néant, Paris, Gallimard, 2008, p. 344.

${ }_{20}$ Ibid., p. 343. À l'opposé, " contre une phénoménologie intransigeante », Ricœur plaide, pour une coordination entre compréhension et explication, J.-P. Changeux, P. Ricœur, La Nature et la règle. Ce qui nous fait penser, op. cit., p. 134.
} 
l'affectivité : alors que l'affectivité originaire, pré-intentionnelle, est la pure conscience du corps en tant qu'être-pour-soi - de cette affectivité relèvent par exemple la douleur pure, la bonne ou la mauvaise humeur comme «tonalité affective non-thétique»-, l'affectivité intentionnelle est déjà une affectivité constituée et suppose la visée $\mathrm{d}^{\prime}$ un objet intentionnel ${ }^{21}$. Ainsi, la haine (ou l'amour) présente un double aspect : elle s'empare de mon être, elle est soufferte, et elle est éclatement, visée intentionnelle d'autrui dont le caractère haïssable est saisi «objectivement», par exemple, à même sa tête qui « ne me revient pas ».

Enfin, troisième distinction, il ne faut pas confondre, d'une part, la haine qui, à l'instar de l'amour, est une entreprise, mais qui, à la différence de l'amour, est animée par le projet spécifique de la destruction d'autrui - pensons par exemple à la manière dont Iago ourdit la mort d'Othello - et, d'autre part, la haine en tant qu'émotion, où la haine prend la forme d'une explosion de haine. Elle est alors une émotion, distincte de la colère ou de la tristesse, et s'accompagne de conduites particulièrement dramatiques, à la fois brèves, spectaculaires et violentes, qui peuvent s'achever dans le sang lorsqu'elles n'empruntent pas la voie d'un assouvissement imaginaire. La haine est ainsi une émotion, c'est-à-dire un vécu de conscience qui se distingue par un ensemble de modifications corporelles et qui fait partie de ces conduites dans lesquelles, nous dit l'Esquisse, il revient au corps de se transformer afin de transformer le monde. Qu'est-ce à dire? Qu'est-ce que l'émotion?

Tout d'abord, l'émotion ne saurait être selon Sartre un ensemble de réactions désordonnées. L'émotion est une conduite qui répond à une finalité ; elle est dite « un système organisé de moyens qui visent une fin » ${ }^{22}$. En outre, l'émotion est une conduite qui, avons-nous dit, vise à transformer le monde. Mais il nous faut immédiatement ajouter un terme décisif. Dans l'émotion, la conscience transforme le monde magiquement. Cette notion de magie est au cœur de la réflexion sartrienne sans être à vrai dire réellement approfondie. Dans l'Esquisse, Sartre renvoie à plus tard l'exploration de ce qu'il tient pour non pas " une qualité éphémère que nous posons sur le monde au gré de nos humeurs » mais pour « une structure existentielle du monde » et, notamment $\mathrm{du}$ monde social ${ }^{23}$. Sans doute, Sartre reprend-il sans le

\footnotetext{
${ }^{21}$ J.-P. Sartre, L'Être et le néant, op. cit., p. 370-371.

22 Esquisse, p. 27.

${ }^{23}$ Sartre soutient même que «l'homme est toujours un sorcier pour l'homme et le monde social est d'abord magique », Esquisse, p. 58.
} 
dire certaines thèses de Lucien Levy-Bruhl sur la mentalité primitive, exposées en 1910 dans son ouvrage intitulé Les fonctions mentales dans les sociétés inférieures. Peut-être même pense-t-il à Auguste Comte et à sa loi des trois états de l'esprit humain.

Quoi qu'il en soit, Sartre semble reconnaitre à l'homme la possibilité d'adopter deux attitudes distinctes par rapport au monde : une attitude magique, qui est dite originelle ${ }^{24}$-, et une attitude pragmatique, rationnelle. Cette dernière repose sur l'intuition pragmatique $\mathrm{du}$ déterminisme du monde - Sartre s'inspire manifestement mais vaguement de la description par Heidegger du monde de la préoccupation avec ses pragmata ou outils (Zeuge) ${ }^{25}$. Ainsi, lorsque nous voulons agir pour satisfaire un besoin ou un désir, certains objets qui nous entourent se présentent comme des moyens pour atteindre nos fins tandis que d'autres semblent des obstacles. Comme l'écrit Sartre, "le monde de nos désirs, de nos besoins et de nos actes apparaît comme sillonné de chemins étroits et rigoureux qui conduisent à tel ou tel but déterminé ». Mais ce monde est difficile et parfois même, trop difficile. Une autre attitude est alors possible : l'attitude magique qui consiste à agir immédiatement sur le monde sans y toucher, c'està-dire sans s'astreindre à enchaîner les moyens disponibles pour atteindre réellement la fin désirée.

Reprenons l'exemple de la peur : « je vois venir vers moi une bête féroce, mes jambes se dérobent sous moi, mon cœur bat plus faiblement, je pâlis, je tombe et je m'évanouis ». Il va de soi qu'il ne $s^{\prime}$ agit pas d'une conduite réflexive qui serait délibérée et qui relèverait de la volonté, mais d'une conduite préréflexive et spontanée ${ }^{26}$. L'émotion en question est ici la peur dite passive, en opposition à la peur active où je fuis en prenant mes jambes à mon coup. Sartre interprète ainsi la conduite de la peur passive :

faute de pouvoir éviter le danger par les voies normales et les enchaînements déterministes, je l'ai nié. J'ai voulu l'anéantir. L'urgence du danger a servi de motif pour une intention annihilante qui a commandé une conduite magique ${ }^{27}$.

D'une manière générale, l'émotion est une conduite incantatoire qui vise à transformer magiquement le monde. Cela est particulièrement manifeste dans la colère lorsque je tempête et frappe tout ce

\footnotetext{
24 Ibid., p. 59.

${ }^{25}$ M. Heidegger, Etre et temps, trad. F. Vezin, Paris, Gallimard, 1986, § 15.

${ }^{26}$ Esquisse, p. 45.

27 Idem.
} 
qui m'entoure. Ce faisant, je tente d'abolir symboliquement, magiquement les chaines qui m'entravent.

\section{La surprise est-elle une émotion?}

Afin de répondre à une telle question, il peut être fructueux d'étudier tout d'abord les gestes et les mimiques de la surprise. Dans ce but, nous pouvons interroger les grands maîtres de l'art dramatique ou encore des arts plastiques qui savent mieux que quiconque comment mettre en scène la surprise. Par exemple, Charles Le Brun, peintre français du XVII e, dans une conférence sur L'expression générale et particulière, dont l'édition est rehaussée de dessins du peintre, nous donne la caractérisation suivante des mouvements du visage dans l'admiration alias la surprise, dont il emprunte la définition à Descartes ${ }^{28}$ :

Comme nous avons dit que l'admiration est la première et la plus tempérée de toutes les passions, et où le cœur sent moins d'agitation, le visage aussi reçoit fort peu de changement en toutes ses parties; et s'il y en a, il n'est que dans l'élévation du sourcil : mais il aura les deux côtés égaux, et l'œil sera un peu plus ouvert qu'à l'ordinaire [...]. La bouche sera aussi entrouverte, mais elle paraitra sans aucune altération, non plus que tout le reste de toutes les autres parties du visage. Cette passion ne produit qu'une suspension de mouvement pour donner le temps à l'âme de délibérer sur ce qu'elle a à faire, et pour considérer avec attention l'objet qui se présente à elle ${ }^{29}$.

Si on veut bien laisser de côté l'interprétation téléologique, d'inspiration également cartésienne de cette passion $^{30}$, une formule doit retenir ici notre attention: "cette passion ne produit qu'une suspension du mouvement ». Elle est donc décrite non pas comme une conduite mais bien plutôt comme son interruption. Dans les cas extrêmes, en effet, la surprise coupe le souffle ; elle abolit la parole en lui substituant parfois un cri ou une interjection; elle paralyse les gestes ainsi que tout mouvement corporel; corrélativement, elle

\footnotetext{
28 "L'admiration est une surprise qui fait que l'âme considère avec attention les objets qui lui semble rares et extraordinaires ", Ch. Le Brun, L'Expression des passions et autres conférences, Paris, Éditions Dédale Maisonneuve et Larose, 1994, p. 56 ; R. Descartes, Les passions de l'âme, Paris, Gallimard, 1969, article 70, p. 77.

${ }^{29} \mathrm{Ch}$. Le Brun, L'Expression des passions et autres conférences, op. cit., p. 66. On pourra noter que le dessinateur Hergé respecte à la lettre les recommandations du peintre. Cf. par exemple la couverture de L'Etoile mystérieuse.

30 Pour Descartes, les passions « sont toutes bonnes de leur nature, et nous n'avons rien à éviter que leurs mauvais usages ou leurs excès ", Traité des passions, article 211.
} 
semble suspendre ou figer le cours du monde. Ainsi la surprise opère-t-elle une sorte d'épochè involontaire. En outre, la surprise ne dure pas - ou presque pas ${ }^{31}$ - et, l'instant de surprise passé, on a vite fait de reprendre ses esprits. Question: cette première description nous permet-elle de tenir la surprise pour une émotion au sens sartrien du terme? S'agit-il d'une conduite et, si tel est le cas, que recherche la conscience et comment? Reconnaissons-le, en raison de sa brièveté et de son caractère suspensif, il semble difficile de tenir la surprise pour une conduite. Dans le cas de la peur passive, nous avons vu que la conscience visait à supprimer le danger grâce à son annihilation magique. Or, dans le cas de la surprise, nous ne trouvons manifestement rien d'analogue.

Cependant, l'Esquisse nous donne une indication précieuse qui permet de considérer la surprise sinon comme une émotion du moins comme un phénomène apparenté, qui pourrait être explicité à partir de ce que Sartre écrit à propos de l'admiration et de l'horreur. Du reste, l'horreur et l'admiration ne sont-elles pas deux modalités, l'une positive, l'autre négative, de la surprise? En tout cas, voici ce que Sartre écrit à ce propos :

Cette théorie de l'émotion [la sienne] n'explique pas certaines réactions brusques d'horreur et d'admiration qui nous saisissent parfois devant des objets apparus tout à coup. Par exemple un visage grimaçant apparaît soudain et se colle à la vitre de la fenêtre ; je me sens envahi de terreur. Ici, évidemment, il n'y a pas de conduite à tenir, il semble que l'émotion n'ait point de finalité. [...] Pourtant, si l'on y réfléchit il s'agit de phénomènes très particuliers mais susceptibles de recevoir une explication qui cadre avec les idées que nous venons d'exposer. Nous avons vu que dans l'émotion, la conscience se dégrade et transforme brusquement le monde déterminé où nous vivons en un monde magique. Mais il y a une réciproque : $c^{\prime}$ est ce monde lui-même qui parfois se révèle à la conscience comme magique au lieu qu'on l'attendait déterminé ${ }^{32}$.

En d'autres termes, Sartre envisage ici des phénomènes comme l'horreur ou l'admiration qui, à première vue, ne s'accordent pas avec sa conception de l'émotion dans la mesure où il ne s'agit pas d'une

\footnotetext{
${ }^{31}$ Natalie Depraz nous met en garde cependant contre le mythe de l'instantanéité de la surprise et développe l'hypothèse que "même la fulgurance prend du temps, qu'elle soit corporelle ou interne ; la surprise est un processus, même s'il s'agit d'un micro-processus ", N. Depraz, "La surprise. Une dynamique circulaire de verbalisation multivectorielle», in N. Depraz et C. Serban, La Surprise à l'épreuve des langues, op. cit., p. 32-33.

${ }^{32}$ Esquisse, p. 57-58. À vrai dire, cette réciproque n'est pas sans mettre en difficulté l'ontologie sartrienne de la conscience : comment le monde peut-il agir sur une conscience qui dans son être échappe à toute action causale?
} 
conduite mais d'une réaction affective dans laquelle, néanmoins, on retrouve la structure existentielle du monde, qu'instaure l'attitude magique. La grande différence avec l'émotion stricto sensu, c'est donc que, dans le cas de l'horreur, "c'est le monde lui-même qui se révèle brusquement comme magique autour de nous " ${ }^{33}$. Par exemple, la manière dont soudain un visage hideux se colle à la fenêtre plonge brusquement la conscience dans un monde magique où, loin d'être tenu à distance par la vitre derrière laquelle il surgit, ce visage semble en liaison immédiate avec notre corps, comme si cet être qui nous horrifie échappait aux exigences spatio-temporelles de l'attitude pragmatique $^{34}$. Essayons d'aborder dans cette perspective la surprise.

\section{Une coupe de cheveux malheureuse}

Dans son autobiographie, Les Mots (1964), que l'on peut également lire comme un essai d'auto-analyse existentielle, Sartre raconte comment son grand-père, à l'insu de toute la famille, emmena un jour son petit-fils, Jean-Paul, chez le coiffeur. Voici le récit de cette surprise :

Mon grand-père s'agaçait de ma longue chevelure : « c'est un garçon, disait-il à ma mère, tu vas en faire une fille; je ne veux pas que mon petit-fils devienne une poule mouillée! » Anne-Marie tenait bon ; elle eût aimé, je pense, que je fusse une fille pour de vrai. [...] Un jour j'avais sept ans - mon grand-père n'y tint plus: il me prit par la main, annonçant qu'il m'emmenait en promenade. Mais, à peine avions-nous tourné le coin de la rue, il me poussa chez le coiffeur en me disant : "Nous allons faire une surprise à ta mère ». J'adorais les surprises. Il y en avait tout le temps chez nous. Cachotteries amusées ou vertueuses, cadeaux inattendus, révélations théâtrales suivies d'embrassements [...] Bref les coups de théâtre faisaient mon petit ordinaire et je regardais avec bienveillance mes boucles rouler le long de la serviette blanche [...] ; je revins glorieux et tondu. Il y eut des cris mais pas d'embrassements et ma mère s'enferma dans sa chambre pour pleurer: on avait troqué sa fillette contre un garçonnet. Il y avait pis : tant qu'elles voltigeaient autour de mes oreilles, mes belles anglaises lui avaient permis de refuser l'évidence de ma laideur [...] Mon grand-père semblait lui-même tout interdit ; on lui avait confié sa petite merveille, il avait rendu un crapaud ${ }^{35}$.

\footnotetext{
${ }^{33}$ Ibid., p. 59.

34 Pour comprendre cette description de l'horreur, pensons au cinéma fantastique et, par exemple, à la manière dont Murnau suscite l'horreur du spectateur dans Nosferatu, le vampire (1922).

35 J.-P. Sartre, Les Mots, Paris, Gallimard, 1972, p. 88-89.
} 
1. Ce qui est tout d'abord frappant dans ce récit, c'est la manière dont Sartre inscrit la surprise dans une culture familiale de l'inattendu, du coup de théâtre, bref d'un quotidien volontiers magique. Ainsi la surprise est-elle une façon d'être ensemble pour la famille Schweitzer et, plus exactement, une conduite ludique, une manière de jouer et de se jouer d'autrui: de jouer parce que ce qui arrive ne comporte aucune espèce de gravité, à moins que la surprise échoue et tourne mal; de se jouer au sens où dans cette entreprise autrui fait l'objet d'une sorte de manipulation pour rire. On peut, à partir de là, se demander si la surprise n'est pas un événement éminemment intersubjectif de sorte que si les choses peuvent nous surprendre en déjouant notre attente, elles ne nous surprennent qu'en un sens dérivé, second, comme si le cours des choses se mettait à imiter les surprises que les hommes s'aménagent. Comme si : car les phénomènes naturels sont habituellement sans surprise. Ils peuvent susciter notre étonnement au sens grec du terme, c'est-à-dire se révéler problématique et susciter notre curiosité mais non pas nous surprendre: nous savons avec Hume et Kant que le soleil chauffe la pierre et qu'il la chauffera demain comme il l'a chauffé hier. Et lorsque du pain tombe du ciel, comme dans l'Exode, il s'agit d'un phénomène évidemment surnaturel qui ne relève donc pas du cours des choses.

2. La surprise décrite par Sartre est une entreprise, c'est-à-dire un projet dont les protagonistes sont, d'une part, ses acteurs (le petit-fils, le grand-père, qui est également auteur et metteur en scène), et, $\mathrm{d}$ 'autre part, ses spectateurs (la grand-mère, Louise Schweitzer, et la mère de Jean-Paul, Anne-Marie Sartre). Et si nous parlons d'acteurs et de spectateurs, c'est afin de souligner la dimension éminemment théâtrale de cette entreprise qui est un spectacle, soigneusement mis en scène dont le dénouement ne doit pas être compromis par une révélation intempestive. $\mathrm{Du}$ reste, ce moment final est explicitement assimilé par Sartre à un coup de théâtre, au sens d'un événement inattendu qui, en l'occurrence, réserve une mauvaise surprise à ses acteurs comme à ses spectateurs.

3. Sartre insiste fortement sur le caractère magique de cette mauvaise surprise en l'assimilant à un tour de prestidigitateur qui aurait mal tourné. Il écrit à propos d'Anne-Marie qui s'en va pleurer dans sa chambre: " on avait troqué sa fillette contre un garçonnet ». Et au sujet du grand père qui demeure interdit : " on lui avait confié sa petite merveille, il avait rendu un crapaud $»^{36}$.

\footnotetext{
${ }^{36} \mathrm{Il}$ est tentant d'établir à ce propos un parallèle avec la coutume d'emballer les cadeaux afin de ménager une surprise à son destinataire. Et même si cela peut paraître futile, on peut remarquer
} 
4. La surprise décrite par Sartre est, en un sens, un échec : «Il y eu des cris et pas d'embrassements et ma mère s'enferma dans sa chambre pour pleurer ». Mais, à vrai dire, l'échec ne concerne pas la surprise elle-même puisque même mauvaise, la surprise reste relativement grande - non pas totale, dans la mesure où il était question de coiffeur depuis quelques jours -, à la mesure, d'une part, de l'ignorance par la grand-mère et la mère de la laideur de l'enfant et, d'autre part, de la soudaineté de la découverte. Nous touchons ici à la dimension temporelle de la surprise, c'est-à-dire de l'événement sans prémices, auquel rien ne nous prépare et qui est d'autant plus surprenant qu'il ne peut être anticipé. La surprise de ce point de vue est totale lorsqu'elle déjoue absolument toute attente. Elle est nulle, en revanche, lorsque l'événement est par trop prévisible.

\section{Conclusion}

S'il est vrai que des émotions comme la colère ou la peur sont bien des conduites préréflexives, involontaires et intentionnelles, la surprise, parce qu'elle ne partage manifestement pas une telle visée, n'est pas à proprement parler une émotion mais bien plutôt, comme l'horreur ou l'admiration, une quasi-émotion. Comme nous l'avons $\mathrm{vu}$, elle est suscitée par le caractère soudainement magique du monde et, plus particulièrement dirions-nous, des relations humaines. L'homme n'est-il pas par excellence celui qui déjoue les attentes de ses semblables, y compris lorsque, tel le Christ, il fait des miracles? En ce sens, comme l'écrit Sartre, "l'homme est toujours un sorcier pour l'homme $»^{37}$. Les choses, en revanche, sont éminemment prévisibles et ne peuvent guère surprendre. C'est du reste ce que laisse entendre Husserl lorsqu'il s'interroge sur la typique de l'aperception de l'expérience naturelle :

quand nous voyons un chien, nous voyons aussitôt par avance sa manière d'être à venir, sa manière typique de manger, de jouer, de courir, de sauter, etc. Nous ne voyons pas actuellement ses dents, mais, bien que nous n'ayons encore jamais vu ce chien, nous savons d'avance à quoi ressemblent ses dents - non dans leur détermination individuelle, mais précisément typique, dans la mesure où nous

que l'emballage ne doit être ni trop fragile ni trop résistant : s'il faut aller chercher des ciseaux à la cuisine, trouver un cutter, etc., l'effet de surprise est raté car on renoue avec une intuition pragmatique du monde. Il faut au contraire que le papier cadeau puisse se déchirer sans effort afin de conférer au surgissement de l'objet offert un caractère magique.

37 Esquisse, p. 58. 
avons déjà eu antérieurement et fréquemment l'expérience d'animaux «semblables », de «chiens », et éprouvé à leur propos qu'ils ont quelque chose comme une «denture», et d'un type de cette sorte $^{38}$.

Il va de soi qu'un chien peut avoir perdu toutes ses dents mais cette possibilité demeure encore dans l'horizon d'attente que prédéfinit la typique de l'expérience naturelle. Un tel chien peut susciter l'étonnement, aiguiser la curiosité mais ne peut pas véritablement surprendre.

Mais nous ne voudrions pas achever notre propos sans soulever une question à laquelle, cependant, il conviendrait d'accorder plus de place. Nous avons, en première partie, adopté une perspective résolument antinaturaliste, convaincu que la psychologie phénoménologique ne devait pas se laisser intimider par les formes plus récentes d'une approche "physicaliste » de phénomènes qui relèvent avant tout de la conscience. Sans doute parvient-on déjà à établir un jeu de correspondances relativement fines entre ce que découvrent, d'une part, la neurobiologie et, de l'autre, la psychologie phénoménologique $^{39}$; mais les résultats n'en demeureront pas moins sur «deux plans incommunicables », comme en témoigne la solution de continuité terminologique entre leurs points de vue. Mais il va de soi que - pensons à Ricœur et à sa conception d'une cause-substrat ou cause matérielle - cet antinaturalisme ainsi que la compréhension sartrienne de l'émotion comme conduite magique sont pour une large part discutables. Dès lors on peut se demander dans quelle mesure notre description est une interprétation et relève d'une herméneutique phénoménologique, dans quelle mesure elle parvient à dévoiler la chose même et ressortit à une phénoménologie pré-herméneutique $^{40}$. Il semble que la surprise renvoie nécessairement à une conscience intentionnelle incarnée, qui tressaille sous le coup de la surprise et dont la pensée est comme suspendue dans son cours; que cette conscience vise nécessairement un événement qui déjoue son anticipation; et que la grandeur ou la force d'une surprise est

\footnotetext{
38 E. Husserl, Expérience et jugement, trad. D. Souche-Dagues, Paris, PUF, 1970, § 83, p. 401.

${ }^{39}$ Dans cette perspective, Étienne Bimbenet note au sujet des neurones miroirs que l'aire F5 du cerveau «redouble au niveau neurophysiologique l'attestation phénoménologique de l'intersubjectivité et de l'inter-corporéité ». Cependant, l'idée d'un redoublement, avec ce qu'elle implique d'identité dans l'altérité, est-elle pertinente en l'occurrence? Le niveau neurophysiologique n'est-il pas tout autre ? É. Bimbenet, Après Merleau-Ponty, Paris, Vrin, 2011, p. 241.

${ }^{40}$ Nous empruntons cette interrogation sur la saisie de la chose même, sa description et son interprétation au livre de Claude Romano, Au cœur de la raison, la phénoménologie, Paris, Gallimard, 2011, p. 897.
} 
nécessairement fonction de son imprévisibilité subjective. Tel serait le noyau eidétique de la surprise. En revanche, que la surprise soit une quasi-émotion, suscitée, à l'instar de l'horreur et de l'admiration, par un monde qui se révèle brusquement comme magique autour de nous, présuppose l'assimilation de l'émotion en général à une conduite magique et semble relativement incertain.

Ainsi, cette brève étude de la surprise nous permet-elle de définir la psycho-phénoménologie comme la science des diverses conduites, au sens large, d'une conscience incarnée dans le monde, fondée sur le dévoilement préalable du mode d'être de l'existence, et dont les connaissances relèvent soit d'une eidétique soit d'une herméneutique phénoménologiques. 